\title{
RUDIN VARIETIES IN PRODUCTS OF TWO ANNULI ${ }^{1}$
}

\author{
SERGIO E. ZARANTONELLO
}

ABSTRACT. Let $Q$ be an annulus and $\partial Q$ its boundary. If $f$ is holomorphic in $Q \times Q$ and its zero set is bounded away from $\partial Q \times \partial Q$, then there exists a bounded holomorphic function $F$ with the same zeros as $f$ such that $F^{-1}$ is bounded near $\partial Q \times \partial Q$.

I. Introduction. Let $\Omega$ be a domain in $\mathrm{C}^{n}$ and $\breve{\partial} \Omega$ be the Šilov boundary of the algebra of holomorphic functions in $\Omega$ with continuous boundary values. A subset $V$ of $\Omega$ is a Rudin variety if it is the zero set of a holomorphic function in $\Omega$, and if none of its limit points lie in $\check{\partial} \Omega$.

It is known [1], [3], that in a polydisc a Rudin variety is necessarily the zero set of a bounded holomorphic function. We show here that this is also the case in a product of two annuli. Unfortunately the method used does not extend to products of more than two annuli.

We use the notation of [1]. The complex numbers will be denoted by $\mathbf{C}$. Let $0<r_{1}<r_{2}<\infty$ and define

$$
\begin{aligned}
Q\left(r_{1}, r_{2}\right) & =\left\{z \in \mathbf{C}: r_{1}<|z|<r_{2}\right\}, \\
\partial Q\left(r_{1}, r_{2}\right) & =\left\{z \in \mathbf{C}:|z|=r_{1} \text { or }|z|=r_{2}\right\} .
\end{aligned}
$$

For any two sets $S_{1} \subset \mathrm{C}$ and $S_{2} \subset \mathrm{C}, S_{1} \times S_{2}$ denotes their cartesian product. Points of $\mathbf{C} \times \mathbf{C}$ will be denoted by $\left(z_{1}, z_{2}\right)$ or simply by $z$. Whenever $\Omega$ is a domain in $\mathbf{C} \times \mathbf{C}, H(\Omega), H^{\infty}(\Omega)$, and $h^{\infty}(\Omega)$ denote the classes of holomorphic, bounded holomorphic, and of holomorphic functions with bounded real parts, respectively. If $f \in H(\Omega)$ its zero set $Z(f)$ is the set $\{z \in \Omega: f(z)=0\}$. Two functions $f_{1}, f_{2}$ in $H(\Omega)$ are said to have the same zeros if their quotient $f_{1} f_{2}^{-1}$ is an invertible holomorphic function in $\Omega$. For any function $f, \operatorname{Re} f$ denotes its real part. Finally, the exponential function will be denoted by exp.

Received by the editors February 27, 1974.

AMS (MOS) subject classifications (1970). Primary 32A30; Secondary 32A10.

Key words and phrases. Rudin variety, multiplicative Cousin problem, holomorphic, annulus, polyannulus.

1 This paper constitutes part of a thesis submitted to the University of Wisconsin. The author would like to thank W. Rudin for supervising the research. 
II. Two lemmas. We start with two lemmas. For the first we state the result and refer the reader to $[1, \mathrm{p} .92]$ for a proof.

Fix $0<r_{1}<r_{2}<\infty$, let $U_{1}=\left\{\lambda \in \mathbf{C}:|\lambda|>r_{1}\right\}$ and $U_{2}=\left\{\lambda \in \mathbf{C}:|\lambda|<r_{2}\right\}$. Let $Q$ be an arbitrary a nnulus centered at the origin.

Lemma 1. Any $g \in h^{\infty}\left(\left(U_{1} \cap U_{2}\right) \times Q\right)$ can be written $g=g_{1}-g_{2}$ where $g_{1} \in h^{\infty}\left(U_{1} \times Q\right)$ and $g_{2} \in h^{\infty}\left(U_{2} \times Q\right)$.

The next lemma proves that a certain type of multiplicative Cousin problem with bounded data can always be solved in a product of two annuli (the solution being a bounded holomorphic function). Without loss of generality the two annuli will be centered at the origin and have the same inner and outer radii, respectively.

Lemma 2. Let $Q$ be an annulus centered at the origin and $\left\{A_{\alpha}\right\}$ a finite covering of $Q \times Q$ consisting of products of annuli centered at the origin. If on each $A_{a}$ we are given a function $f_{\alpha} \in H^{\infty}\left(A_{\alpha}\right)$, and for all a and $\beta, f_{\alpha} f_{\beta}^{-1}$ is invertible in $H^{\infty}\left(A_{\alpha} \cap A_{\beta}\right)$, there exists a function $F \in H^{\infty}(Q \times Q)$ such that for every $\alpha, F f_{\alpha}^{-1}$ is invertible in $H^{\infty}\left(A_{\alpha}\right)$.

Proof. Let $Q=Q(r, r+\epsilon)$ and define

$$
Q_{1}=Q(r, r+2 \epsilon / 3), \quad Q_{2}=Q(r+\epsilon / 3, r+\epsilon) .
$$

Suppose the induced "Cousin problem" can be solved in both $Q_{1} \times Q$ and $Q_{2} \times Q$ with solutions $F_{1}$ and $F_{2}$, respectively. Since for each $\alpha$, $F_{1} f_{\alpha}^{-1}$ and $f_{a} F_{2}^{-1}$ are invertible in $H^{\infty}\left(A_{\alpha} \cap\left(\left(Q_{1} \cap Q_{2}\right) \times Q\right)\right), F_{1} F_{2}^{-1}$ will be invertible in $H^{\infty}\left(\left(Q_{1} \cap Q_{2}\right) \times Q\right)$.

Fix $r+\epsilon / 3<\rho<r+2 \epsilon / 3$, and for each $t \in[0,1]$ define $\Gamma_{1}(t)=$ $\left(\rho e^{2 \pi i t}, \rho\right)$ and $\Gamma_{2}(t)=\left(\rho, \rho e^{2 \pi i t}\right)$. Clearly $\Gamma_{1}$ and $\Gamma_{2}$ constitute a basis for the fundamental group of $\left(Q_{1} \cap Q_{2}\right) \times Q$. Let $-\alpha_{1}$ and $-\alpha_{2}$ be the indices of $\left(F_{1} F_{2}^{-1}\right) \circ \Gamma_{1}$ and $\left(F_{1} F_{2}^{-1}\right) \circ \Gamma_{2}$ (both of which are loops in $\mathbf{C}-\{0\}$ ), and define

$$
G(z)=F_{1}(z) F_{2}(z)^{-1} z_{1}^{a}{ }_{1}^{a} z_{2}^{2} \quad\left(z \in\left(Q_{1} \cap Q_{2}\right) \times Q\right) .
$$

It follows that $G \circ \Gamma$ has index 0 for every loop $\Gamma$ in $\left(Q_{1} \cap Q_{2}\right) \times Q$, therefore there exists $g \in H\left(\left(Q_{1} \cap Q_{2}\right) \times Q\right)$ such that

$$
G(z)=\exp g(z) \quad\left(z \in\left(Q_{1} \cap Q_{2}\right) \times Q\right) .
$$

Since $G$ is invertible in $H^{\infty}\left(\left(Q_{1} \cap Q_{2}\right) \times Q\right)$, Re $g$ must be bounded. Let $U_{1}=\{\lambda \in \mathrm{C}:|\lambda|>r+\epsilon / 3\}$ and $U_{2}=\{\lambda \in \mathrm{C}:|\lambda|<r+2 \epsilon / 3\} ; g$ is in 
$h^{\infty}\left(\left(Q_{1} \cap Q_{2}\right) \times Q\right)$ and $U_{1} \cap U_{2}=Q_{1} \cap Q_{2}$, so by Lemma $1 g=g_{1}-g_{2}$ with $g_{1} \in h^{\infty}\left(U_{1} \times Q\right)$ and $g_{2} \in h^{\infty}\left(U_{2} \times Q\right)$. Hence (1) can be written as

$$
\exp \left(g_{1}(z)-g_{2}(z)\right)=F_{1}(z) F_{2}(z)^{-1} z_{1}^{a}{ }_{1} z_{2}^{a_{2}} \quad\left(z \in\left(Q_{1} \cap Q_{2}\right) \times Q\right)
$$

which enables us to define a function $F \in H^{\infty}(Q \times Q)$ by

$$
F(z)= \begin{cases}F_{1}(z) \exp \left(-g_{1}(z)\right) z_{1}{ }_{1}{ }^{a} z_{2}{ }^{a} & \text { if } z \in Q_{2} \times Q, \\ F_{2}(z) \exp \left(-g_{2}(z)\right) & \text { if } z \in Q_{1} \times Q .\end{cases}
$$

It is easy to see that for each $\alpha, F f_{a}^{-1}$ is invertible in $H^{\infty}\left(A_{a}\right)$, so if the induced "Cousin problem" can be solved in both $Q_{1} \times Q$ and $Q_{2} \times Q$ it can also be solved in $Q \times Q$.

Suppose now that our lemma is false. By what we just mentioned the induced "Cousin problem" cannot be solved in both $Q_{1} \times Q$ and $Q_{2} \times Q$; suppose it cannot be solved in $Q_{1} \times Q$. Let $Q_{1}^{1}=Q_{1} \times Q, Q_{2}^{1}=Q_{1} \times Q_{1}, Q_{2}^{2}$ $=Q_{1} \times Q_{2}$. Arguing as before, the induced "Cous in problem" would not be solvable in both $Q_{2}^{1}$ and $Q_{2}^{2}$, so on one of these, call it $Q_{2}^{k}$, it will be unsolvable. Iterating this procedure, proceeding cyclicly through the complex coordinates $z_{1}$ and $z_{2}$, we obtain a nested sequence

$$
Q_{1}^{1} \supset Q_{2}^{k} \supset Q_{3}^{k} \supset \ldots \supset Q_{m}^{k}{ }_{m} \supset \ldots
$$

of products of annuli centered at the origin whose thinness eventually decreases to zero, on none of which we are able to solve the induced problem. Since $\left\{A_{\alpha}\right\}$ is a finite collection of products of annuli centered at the origin and $Q \times Q=\bigcup A_{\alpha}$, for some integer $m$ and some $\alpha$ we will have $Q_{m}^{k} \mathrm{C}$ $A_{a}$. But $f_{a}$ solves the induced "Cousin problem" in $A_{a}$ (and hence in $Q_{m}^{k}$ ) so we have a contradiction. Consequently the lemma is true.

III. Rudin varieties in a product of two annuli. Fix $0<r_{1}<r_{2}<\infty$ and let $Q=Q\left(r_{1}, r_{2}\right), \partial Q=\partial Q\left(r_{1}, r_{2}\right)$.

Theorem 1. Let $f \in H(Q \times Q)$. If $Z(f)$ has no limit points in $\partial Q \times \partial Q$ then there exists $F \in H^{\infty}(Q \times Q)$ with the same zeros as $F$, moreover $F^{-1}$ is bounded near $\partial Q \times \partial Q$.

Proof. Fix $0<\epsilon<\left(r_{2}-r_{1}\right) / 2$ sufficiently small so that

$$
\left(Q\left(r_{1}, r_{1}+\epsilon\right) \cup Q\left(r_{2}-\epsilon, r_{2}\right)\right) \times\left(Q\left(r_{1}, r_{1}+\epsilon\right) \cup Q\left(r_{2}-\epsilon, r_{2}\right)\right)
$$

does not intersect $Z(f)$, and define 


$$
Q_{1}=Q\left(r_{1}, r_{1}+\epsilon / 2\right), \quad Q_{2}=Q\left(r_{2}-\epsilon / 2, r_{2}\right)
$$

For each $z_{1} \in Q_{1}\left(r_{1}, r_{2}+\epsilon\right)$ the function $z_{2} \rightarrow f\left(z_{1}, z_{2}\right)$ has finitely many zeros, all in $r_{1}+\epsilon<\left|z_{1}\right|<r_{2}-\epsilon$. Let $D_{2}$ denote differentiation $w i t h$ respect to $z_{2}$ and for each $k=0,1,2, \ldots$ define

$$
S_{k}\left(z_{1}\right)=\frac{1}{2 \pi i}\left(\int_{\gamma_{1}}+\int_{\gamma_{2}}\right) \frac{D_{2} f\left(z_{1}, \xi\right)}{f\left(z_{1}, \xi\right)} \xi^{k} d \xi
$$

where

$$
\gamma_{1}(t)=\left(r_{1}+\epsilon / 2\right) e^{-2 \pi i t}, \quad \gamma_{2}(t)=\left(r_{2}-\epsilon / 2\right) e^{2 \pi i t} \quad(t \in[0,1]) .
$$

Note that each $S_{k}\left(z_{1}\right)$ is holomorphic in $Q_{1}$, and that by the residue theorem if $a_{1}\left(z_{1}\right), a_{2}\left(z_{1}\right), \cdots, a_{p}\left(z_{1}\right)$ are the zeros of $z_{2} \rightarrow f\left(z_{1}, z_{2}\right)$ then $S_{k}\left(z_{1}\right)$ $=\sum_{i=1}^{p} a_{i}\left(z_{1}\right)^{k}$. Since $S_{0}\left(z_{1}\right)$ is the number of zeros of $z_{2} \rightarrow f\left(z_{1}, z_{2}\right)$ (counted with their multiplicities), it is an integer valued holomorphic and therefore constant function, i.e. $S_{0}\left(z_{1}\right)=p$ for all $z_{1} \in Q_{1}$.

For each $z_{1} \in Q_{1}$ and all $z_{2}$ define

$$
\phi_{11}(z)=\prod_{i=1}^{p}\left(z_{2}-\alpha_{i}\left(z_{1}\right)\right)=z_{2}^{p}+b_{1}\left(z_{1}\right) z_{2}^{p-1}+\cdots+b_{p}\left(z_{1}\right) .
$$

The connection between the functions $b_{j}$ and $S_{j}$ is given by Newton's identities

$$
b_{j}\left(z_{1}\right)=-\frac{-1}{j}\left(S_{j}\left(z_{1}\right)+S_{j-1}\left(z_{1}\right) b_{1}\left(z_{1}\right)+\cdots+S_{1}\left(z_{1}\right) b_{j-1}\left(z_{1}\right)\right)
$$

for $1 \leq j \leq p$ (see for instance [1, pp. 11, 12]), which show that the coefficients $b_{j}$ are holomorphic in $Q_{1}$, and hence that $\phi_{11}$ is holomorphic in $Q_{1} \times \mathbf{C}$. Also

$$
(\epsilon / 2)^{p}<\left|\phi_{11}(z)\right|<\left(2 r_{2}\right)^{p} \quad \text { if } z \in Q_{1} \times\left(Q_{1} \cup Q_{2}\right) .
$$

If we define $h(z)=F(z) / \phi(z)$ then $h(z)$ has no zeros and is holomorphic in $z_{2} ;$ thus

$$
h(z)=\frac{1}{2 \pi i}\left(\int_{\gamma^{1}}+\int_{\gamma^{2}}\right) \frac{F\left(z_{1}, \xi\right)}{\phi_{11}\left(z_{1}, \xi\right)} \frac{d \xi}{\xi-z_{2}} \quad\left(r_{1}+\frac{\epsilon}{2}<\left|z_{2}\right|<r_{2}-\frac{\epsilon}{2}\right),
$$

which shows that $h(z)$ is holomorphic in $z_{1}$, and hence holomorphic in $Q_{1} \times Q$. We have $\phi_{11} \in H^{\infty}\left(Q_{1} \times Q\right)$, and similarly define $\phi_{12} \in H^{\infty}\left(Q_{2} \times Q\right), \phi_{21} \epsilon$ $H^{\infty}\left(Q \times Q_{1}\right), \phi_{22} \in H^{\infty}\left(Q \times Q_{2}\right)$. All of them recapture the zeros of $f$ in their respective domains, and the quotient of any two, wherever it can be consid- 
ered, is an invertible bounded holomorphic function.

Define now $Q_{3}=Q\left(r_{1}+\epsilon / 3, r_{2}-\epsilon / 3\right)$ and denote the restriction of $f$ to $Q_{3} \times Q_{3}$ by $\Psi$. Then, by what we have said above, $\phi_{11}, \phi_{12}, \phi_{21}, \phi_{22}$ and $\Psi$, and their domains, satisfy the same properties as the functions $f_{a}$ and the sets $V_{\alpha}$ of Lemma 3. Thus there exists a function $F \in H^{\infty}(Q \times Q)$ such that $\phi_{11} F^{-1}, \phi_{12} F^{-1}, \phi_{21} F^{-1}, \phi_{22} F^{-1}$ and $\Psi F^{-1}$ are invertible bounded holomorphic functions. Therefore $F$ has the same zeros as $f$, and since $\phi_{11}$ is bounded away from zero in $Q_{1} \times\left(Q_{1} \cup Q_{2}\right), F^{-1}$ is bounded there. Similarly $F^{-1}$ is bounded in $Q_{2} \times\left(Q_{1} \cup Q_{2}\right)$. The theorem then is proved since we have shown the existence of $F \in H^{\infty}(Q \times Q)$ with the same zero as $f$, and that $F^{-1}$ is bounded in $\left(Q_{1} \cup Q_{2}\right) \times\left(Q_{1} \cup Q_{2}\right)$.

IV. Comments. From Theorem 1 it follows that in $Q \times Q$ a Rudin variety is the zero set of a bounded holomorphic function. For higher complex dimension Lemma 3 still holds, but the Weierstrass polynomials (which can be constructed exactly the same as in Theorem 1), together with $f$ restricted to a "smaller" polyannulus, do not constitute appropriate data for Lemma 3, in the sense that their domains do not cover all of the original polyannulus.

For a polydisc the analogue to Theorem 1 (proved for arbitrary complex dimension) is due to W. Rudin [1], [3]. For complex dimension two a similar scheme to the one above can be used to prove this result, the solvability of the second Cousin problem with bounded data for a polydisc (proved by E. Stout in [4]) playing the role of Lemma 3. This is easy to see, but as with a product of annuli, this method does not appear to be applicable in higher complex dimension.

\section{REFERENCES}

1. W. Rudin, Function theory in polydiscs, Benjamin, New York, 1969. MR 41 $\# 501$.

2. - Real and complex analysis, McGraw-Hill, New York, 1966. MR 35 $\# 1420$.

3. - Zero-sets in polydiscs, Bull. Amer. Math. Soc. 73 (1967), 580-583. MR $35 \# 1819$.

4. E. L. Stout, The second Cousin problem with bounded data, Pacific J. Math. 26 (1968), 379-387. MR $38 \# 3467$.

DEPARTMENT OF MAT HEMATICS, UNIVERSITY OF FLORIDA, GAINESVILLE, FLORIDA 32611 